\title{
Mixture effects of 30 environmental contaminants on incident metabolic syndrome-A prospective study
}

\author{
Lars Lind $^{\mathrm{a}}$, Samira Salihovic ${ }^{\mathrm{b}}$, Erik Lampa ${ }^{\mathrm{c}}$, P. Monica Lind ${ }^{\mathrm{d}, *}$ \\ a Department of Medical Sciences, Cardiovascular Epidemiology, Uppsala University, Uppsala, Sweden \\ b Department of Medical Sciences, Molecular Epidemiology and Science for Life Laboratory, Uppsala University, Uppsala, Sweden \\ c Uppsala Clinical Research Center (UCR), Uppsala, Sweden \\ d Department of Medical Sciences, Occupational and Environmental Medicine, Uppsala University Uppsala, Sweden
}

\section{A R T I C L E I N F O}

\section{Keywords:}

Environmental contaminants

Mixture

Gradient-boosted Classification and Regression Trees (CART)

Metabolic syndrome (MetS)

Epidemiology

Prospective

\begin{abstract}
A B S T R A C T
Background: Several cross-sectional studies have linked different environmental contaminants to the metabolic syndrome (MetS). However, mixture effects have not been investigated and no prospective studies exist regarding environmental contaminants and the MetS.

Objectives: To study mixture effects of contaminants on the risk of incident MetS in a prospective fashion.

Methods: Our sample consisted of 452 subjects from the Prospective Study of the Vasculature in Uppsala Seniors (PIVUS) study (50\% women, all aged 70 years) free from the MetS at baseline, being followed for 10 years. At baseline, 30 different environmental contaminants were measured; 6 polychlorinated biphenyls (PCBs), 3 organochlorine (OC) pesticides, one dioxin, one polybrominated diphenyl ether (all in plasma), 8 perfluoroalkyl substances (in plasma) and 11 metals (in whole blood). The MetS was defined by the ATPIII/NCEP criteria. Gradient boosted Classification and Regression Trees (CARTs) was used to evaluate potential synergistic and additive mixture effects on incident MetS.

Results: During 10-year follow-up, 92 incident cases of the MetS occurred. PCB126, PCB170, hexachlorobenzene (HCB) and PCB118 levels were all associated with incident MetS in an additive fashion (OR 1.73 for a change from 10th to 90th percentile (95\%CI 1.24-3.04) for PCB126, OR $0.63(0.42-0.78)$ for PCB170, OR 1.44 (1.09-2.20) for HCB and OR 1.46 (1.13-2.43) for PCB118). No synergistic effects were found.

Conclusion: A mixture of environmental contaminants, with PCB126, PCB170, HCB and PCB118 being the most important, showed associations with future development of the MetS in an additive fashion in this prospective study. Thus, mixture effects of environmental contaminants could contribute to the development of cardiometabolic derangements.
\end{abstract}

\section{Introduction}

The metabolic syndrome (MetS) is a term used to define subjects with multiple cardiovascular (CV) risk factors, like hypertension, diabetes/impaired glucose tolerance, visceral obesity, low HDL-cholesterol and high serum triglycerides. The MetS was described in the late 1980s by different groups (Reaven, 1988) (Lind et al., 1988), and the clustering of the $\mathrm{CV}$ risk factors have been suggested to be due to visceral obesity and/or insulin resistance. Having the MetS approximately doubles your risk of future CV disease (Sundstrom et al., 2006).

In the last decade it has become apparent that environmental contaminants could play a role in the development of cardio-metabolic diseases (Lind and Lind, 2012; Lind et al., 2016). A number of environmental contaminants have been linked to obesity, diabetes, hypertension and disturbances in lipid metabolism (Goncharov, Haase et al., 2008, Gaskins and Schisterman, 2009, Lee et al., 2011, Lee et al., 2012, Lind et al., 2012, Patel et al., 2012, Fisher et al., 2013, Lind, Zethelius et al. 2014, Lind, Penell et al. 2014, Penell et al., 2014), as well as to the metabolic syndrome in itself (Lee et al., 2007; Uemura et al., 2009; Park et al., 2010; Teppala et al., 2012; Fisher et al., 2013; Lind et al., 2013).

However, previous reports on a link between environmental contaminants and the MetS have used a cross-sectional design, which has the possibility of being biased by reverse causation. Furthermore, these studies have not taken into account that multiple environmental contaminants interfere with each other in the population setting; in previous studies the contaminants are usually evaluated one by one.

In the present study, we addressed some of these shortcomings of

\footnotetext{
* Corresponding author at: Department of Medical Sciences, Occupational and Environmental Medicine, Uppsala University, 75185 Uppsala, Sweden

E-mail addresses: lars.lind@medsci.uu.se (L. Lind), samira.salihovic@medsci.uu.se (S. Salihovic), erik.lampa@ucr.uu.se (E. Lampa), monica.lind@medsci.uu.se (P.M. Lind).
} 
previous investigations in that we studied the effect of a mixture of environmental contaminants on incident MetS in a prospective fashion. We used the Prospective Study of the Vasculature in Uppsala Seniors (PIVUS) study (Lind et al., 2005), in which we have evaluated 30 different environmental contaminants and have followed the cohort for 10 years regarding the development of the MetS. The hypothesis tested was that several contaminants would be associated with incident MetS, either in an additive or a synergistic fashion. We tested this hypothesis by using gradient-boosted CARTs, a statistical method previously deemed suitable for studies of mixture effects of environmental contaminants (Lampa et al., 2014).

\section{Material and methods}

\subsection{Subjects}

Eligible subjects were all aged 70 and lived in the city of Uppsala, Sweden, a city of 185,000 inhabitants. The subjects were randomly chosen from the register of community living. A total of 1016 subjects participated, entailing a participation rate of $50.1 \%$. The prevalence of the MetS at baseline was $23 \%$ in the total cohort. These subjects were invited to a follow-up examination 10 years later, and 606 individuals participated in this follow-up. Following exclusion of 154 subjects who showed the MetS at baseline, 452 subjects were included in the final analyses (being free from the MetS at baseline and participating in the 10-year follow-up).

The study was approved by the Ethics Committee of Uppsala University, and all the participants gave their informed consent prior to the study.

All subjects were investigated in the fasting state in the morning after an overnight fast. Between 8 and 10 am venous samples of whole blood, serum and plasma were collected. The whole blood and plasma samples were collected in EDTA-tubes, while serum was collected in tubes free from additives. After approximately $1 \mathrm{~h}$, the serum was removed to plastic tubes free from additives and the samples were put into a $-80 \mathrm{C}$ freezer. The plasma tubes were spinned and plasma was removed to plastic tubes free from additives and the samples were put into a $-80 \mathrm{C}$ freezer within $1 \mathrm{~h}$. Whole blood was removed from the EDTA-tubes to plastic tubes free from additives and the samples were put into a $-80 \mathrm{C}$ freezer within $1 \mathrm{~h}$. The samples were kept in $-80 \mathrm{C}$ until analysis. The samples were later shipped to different laboratories using dry ice to keep them in the frozen state.

No medication or smoking was allowed after midnight. The participants were asked to answer a questionnaire about their medical history, smoking habits and regular medication.

Blood pressure was measured three times by a calibrated mercury sphygmomanometer to the nearest $\mathrm{mmHg}$ following at least $30 \mathrm{~min}$ of rest. The mean of these three recordings were used. Lipid variables and fasting blood glucose were measured by standard laboratory techniques the same day using unfrozen samples (Carlsson et al., 2010).

These examinations were performed in the same fashion both at age 70 and at age 80 years.

Basic characteristics are presented in Table 1 in the Results section.

Results of the measurements of the 30 environmental contaminants at age 70 are shown in Table 2 in the Results section. The collection of blood samples for the measurements of the environmental contaminants and the other examinations at age 70 was performed on the same day.

\subsection{Chemical analysis}

\subsubsection{Metals}

All 11 metal elements in this study were determined in whole blood at a commercial laboratory (ALS Scandinavia, Luleå, Sweden). The analysis was performed using inductively coupled plasma-sector field mass spectrometry, ICP-SFMS, after microwave-assisted digestion with
Table 1

Means (and SD) or proportions (in \%) for major cardiovascular risk factors at age 70 and 80 years in the sample. $n=452$. $N E=$ not evaluated. For more detailed definitions of exercise levels, please see the Methods section.

\begin{tabular}{|c|c|c|}
\hline & Age 70 & Age 80 \\
\hline Variable & Mean (SD) & Mean (SD) \\
\hline Systolic blood pressure (mm Hg) & $147.1(22.4)$ & $145.7(19.1)$ \\
\hline Diastolic blood pressure (mm Hg) & $77.9(9.8)$ & $73.5(8.9)$ \\
\hline Fasting blood glucose $(\mathrm{mmol} / \mathrm{L})$ & $5.0(0.8)$ & $5.1(1.1)$ \\
\hline Waist circumference $(\mathrm{cm})$ & $89.1(10)$ & $94.6(11.1)$ \\
\hline HDL-cholesterol (mmol/L) & $1.6(0.4)$ & $1.4(0.4)$ \\
\hline Serum triglycerides (mmol/L) & $1.1(0.4)$ & $1.1(0.5)$ \\
\hline LDL-cholesterol (mmol/L) & $3.4(0.8)$ & $3.2(0.9)$ \\
\hline Current smoking (\%) & 8.4 & 3.4 \\
\hline Education level & $\begin{array}{c}<10 \text { years: } 51 \% \\
\text { 10-12 years: } 19 \% \\
>12 \text { years: } 30 \%\end{array}$ & $\mathrm{NE}$ \\
\hline Exercise habits (per week) & $\begin{array}{l}\text { Sedentary: } 9 \% \\
\text { Light exercise only: } 56 \% \\
\text { 1-2 times heavy exercise per } \\
\text { week: } 27 \% \\
>2 \text { times heavy exercise per } \\
\text { week: } 8 \%\end{array}$ & NE \\
\hline Alcohol intake (g/day) & $7.6(8.1)$ & NE \\
\hline Energy intake (kcal/day) & $1921(481)$ & $\mathrm{NE}$ \\
\hline $\begin{array}{l}\text { Fat intake (\% of total energy } \\
\text { intake) }\end{array}$ & 32 & $\mathrm{NE}$ \\
\hline Metabolic syndrome (\%) & 0 & 20 \\
\hline Antihypertensive treatment (\%) & 22 & 55 \\
\hline
\end{tabular}

Table 2

Medians and 10th and 90th percentile for the 30 evaluated environmental contaminants in the present sample $(n=452)$. Wet-weight data are given.

\begin{tabular}{|c|c|}
\hline Variable & $\begin{array}{l}\text { Median (10th and 90th } \\
\text { percentile) }\end{array}$ \\
\hline PCB-118 (2,3',4,4',5-pentachlorobiphenyl) (pg/mL) & $197.2(92,364.2)$ \\
\hline PCB-126 (3,3',4,4',5-pentachlorobiphenyl) (pg/mL) & $39.8(12.4,112.6)$ \\
\hline $\begin{array}{l}\text { PCB-153 (2,2',4,4',5,5'-hexachlorobiphenyl) (pg/ } \\
\quad \mathrm{mL})\end{array}$ & $1394(809,2289.8)$ \\
\hline PCB-169 $\left(3,3^{\prime}, 4,4^{\prime}, 5,5^{\prime}\right.$-hexachlorobiphenyl (pg/mL) & $173(102.6,289)$ \\
\hline $\begin{array}{l}\text { PCB-170 }\left(2,2^{\prime}, 3,4,4^{\prime}, 5,5^{\prime} \text {-heptachlorobiphenyl) (pg/ }\right. \\
\quad \mathrm{mL})\end{array}$ & $494(302.2,787.6)$ \\
\hline $\begin{array}{l}\text { PCB-209 }\left(2,2^{\prime}, 3,3^{\prime}, 4,4^{\prime}, 5,5^{\prime}, 6,6^{\prime}-\right. \\
\text { decachlorobiphenyl) (pg/mL) }\end{array}$ & $26.6(14.6,44.8)$ \\
\hline HCB (hexachlorobenzene) (pg/mL) & $245.4(145.2,424.8)$ \\
\hline OCDD (octachlorodibenzo-p-dioxin) (pg/mL) & $2.4(1.3,5.8)$ \\
\hline TNC (trans-nonachlor) (pg/mL) & $132.4(60,270.8)$ \\
\hline $\begin{array}{l}p, p^{\prime}-\mathrm{DDE}(\mathrm{pg} / \mathrm{mL})(2,2 \text {-bis (4-chlorophenyl)-1,1- } \\
\text { dichloroethene) }\end{array}$ & $1613.6(475.2,5265.8)$ \\
\hline BDE-47 (bromodiphenyl ether) (pg/mL) & $12.6(9,32.4)$ \\
\hline PFHpA (perfluoroheptanoic acid) (ng/mL) & $0.1(0,0.1)$ \\
\hline PFHxS (perfluorohexane sulfonic acid) (ng/mL) & $2.2(1.2,8.5)$ \\
\hline $\begin{array}{l}\text { L-PFOS (linear-perfluorooctane sulfonic acid isomer) } \\
\quad \text { (ng/mL) }\end{array}$ & $13.8(7.7,25.4)$ \\
\hline PFOA (perfluorooctanoic acid) (ng/mL) & $3.5(2,5.7)$ \\
\hline PFNA (perfluorononanoic acid) (ng/mL) & $0.7(0.4,1.4)$ \\
\hline PFDA (perfluorodecanoic acid) (ng/mL) & $0.3(0.2,0.6)$ \\
\hline PFOSA (perfluorooctane sulfonamide) (ng/mL) & $0.1(0.1,0.3)$ \\
\hline PFUnDA (perfluoroundecanoic acid) (ng/mL) & $0.3(0.1,0.5)$ \\
\hline $\mathrm{Al}$ (aluminium) $(\mu \mathrm{mol} / \mathrm{L})$ & $0.6(0.4,1)$ \\
\hline Cd (cadmium) (nmol/L) & $2.3(1.3,5.1)$ \\
\hline Co (cobalt) (nmol/L) & $1.4(0.8,2.8)$ \\
\hline $\mathrm{Cr}$ (chromium) (nmol/L) & $11.7(8.1,29.4)$ \\
\hline $\mathrm{Cu}$ (copper) (nmol/L) & $12.6(10.6,15.3)$ \\
\hline $\mathrm{Hg}$ (mercury) (nmol/L) & $9.7(4.1,22.4)$ \\
\hline Mn (manganese) (nmol/L) & $138.5(93.7,200)$ \\
\hline Mo (molybdenum) (nmol/L) & $9.7(6.6,15.3)$ \\
\hline Ni (nickel) (nmol/L) & $89.4(15.7,429)$ \\
\hline $\mathrm{Pb}$ (lead) $(\mu \mathrm{mol} / \mathrm{L})$ & $0.1(0,0.1)$ \\
\hline Zn (zink) $(\mu \mathrm{mol} / \mathrm{L})$ & $95.5(78,113)$ \\
\hline
\end{tabular}


nitric acid (Rodushkin et al., 2000) according to a method accredited for 10 of the 11 metal elements tested, with $\mathrm{Al}$ being unaccredited. All testing followed the stringent $\mathrm{QA} / \mathrm{QC}$ demands required by the Swedish National Accreditation Body, SWEDAC, in accordance with ISO 17025.

The performing laboratory, ALS Scandinavia $\mathrm{AB}$ in Luleå Sweden, is registered with the FDA and has a certificate of GMPcompliance issued by the Swedish Medical Products Agency.

\subsubsection{Persistent organic pollutants POPs}

POPs were measured in stored plasma samples collected at baseline. Analyses of POPs were performed using a Micromass Autospec Ultima (Waters, Milford, MA, USA) high resolution chromatography coupled to a high resolution mass spectrometry (HRGC/HRMS) system based on the method by Sandau et al. (2003) with some modifications. A total of 23 POPs were measured: 16 polychlorinated biphenyl (PCB) congeners, 5 organochlorine (OC) pesticides, 1 octachlorodibenzo-p-dioxin (OCDD), and 1 polybrominated diphenyl ether (PBDE) congener. Amongst the 23 POPs measured, 2 OC pesticides (trans-chlordane and cis-chlordane) with detection rates $<10 \%$ were not included in the final statistical analyses; the minimum detection rate was $72 \%$ and was observed for BDE47. The recovery of internal standards used for quantification of POPs ranged between $46 \%$ and $110 \%$, and the reproducibility was, for most compounds, below $25 \%$ relative standard deviation (RSD) except for two OC pesticides and one dioxin. Method detection limits ranged between 0.8 and $117.7 \mathrm{pg} / \mathrm{mL}$ depending on the analyte. Further details on POP analyses including quality control are provided elsewhere (Salihovic et al., 2012). Wet-weight plasma concentrations were used in the analyses (and lipids were used in the model to adjust for the lipid dependence of the lipid-soluble POPs).

\subsubsection{Perfluoroalkyl substances PFASs}

The analytical method used to determine plasma concentrations of perfluoroalkyl substances (PFASs) in the samples was successfully validated in terms of recovery, accuracy, and precision (Salihovic et al., 2013; Salihovic et al., 2015).

Briefly, $150 \mu \mathrm{L}$ plasma samples were subjected to protein precipitation using Ostro 96-well plates (Waters Corporation, Milford, MA, USA). Sample extracts were analyzed using an Acquity UPLC ultraperformance liquid chromatography coupled to a Quattro Premier XE HPLC-MS/MS system (Waters Corporation, Milford, MA, USA). Eight PFASs were determined: perfluoroheptanoic acid (PFHpA), perfluorooctanoic acid PFOA, perfluorononanoic acid (PFNA), perfluorodecanoic acid (PFDA), perfluoroundecanoic acid (PFUnDA), perfluorohexane sulfonic acid (PFHxS), linear isomer of perfluorooctane sulfonic acid (L-PFOS), and perfluorooctane sulfonamide (PFOSA). The method detection limits ranged between 0.01 and $0.17 \mathrm{ng} / \mathrm{mL}$ depending on the analyte.

Medians and 10th and 90th percentile of the eight different PFASs with detectable levels $(\mathrm{ng} / \mathrm{mL})$ in $>75 \%$ of the subjects in the sample are given in Table 2 together with data on POPs and metals.

\subsection{Definition of the metabolic syndrome}

The MetS was defined by the The National Cholesterol Education.

Program (NCEP) Expert Panel on Detection, Evaluation, and Treatment of High.

Blood Cholesterol in Adults (Adult Treatment Panel III) (NCEP/ATP III) criteria. Three of the following five criteria should be fulfilled: Blood pressure $>130 / 85 \mathrm{~mm} \mathrm{Hg}$ or antihypertensive treatment, fasting blood glucose $>5.6 \mathrm{mmol} / \mathrm{L}$, serum triglycerides $>1.7 \mathrm{mmol} /$ $\mathrm{L}$, waist circumference $>102 \mathrm{~cm}$ in men and $>88 \mathrm{~cm}$ in women, HDL-cholesterol $<1.0 \mathrm{mmol} / \mathrm{L}$ in men and $<1.3$ in women.

\subsection{Definition of lifestyle factors}

Life-style factors were evaluated by a questionnaire. Educational level was divided into three groups: $<9$ years, 9-12 years and $>12$ years of education. Exercise habits were divided into four groups: $<2$ times light exercise (no sweat) per week, $\geq 2$ times light exercise per week, 1-2 times heavy exercise (sweat) per week, $>2$ times heavy exercise (sweat) per week.

Each participant recorded his or her food consumption during 7 consecutive days using a pre-coded food diary after instructions from a dietician. The pre-coded food diary had been prepared, validated and previously used by the Swedish National Food Administration (NFA) and Statistics Sweden in a food survey of 3000 households in 1989 (Administration, 1994; Becker, 2001). In this study we use data on total energy intake, fat intake and alcohol intake.

\subsection{Statistical analysis}

We have previously shown that certain PCBs cluster together both in the PIVUS study and in the NHANES study (Lampa et al., 2012). Therefore, we only used 6 (PCB118, PCB126, PCB153, PCB169, РCB170 and PCB209) out of the 16 measured PCBs in the present analyses. Those 6 PCBs will cover the main variance in all the measured PCBs.

Gradient-boosted Classification and Regression Trees (CART) were used to investigate the associations between the studied variables and incident MetS. A CART partitions the feature space into disjoint regions with constant predictions to form prediction rules. These prediction rules are created by using binary splits on the input variables and while they may be easily interpreted, the predictive power of single CARTs is usually poor. CARTs are described by their depth which determines the maximum interaction order. A CART containing a single split is called a stump and allows no interactions. A depth 2 CART can contain two-way interactions or a non-linear relationship obtained by successive splits on a variable. A depth three CART can contain at most third order interactions and so on.

Gradient boosting is a technique to improve the poor predictive performance of single CARTs by combining many CARTs into an ensemble. The boosting algorithm briefly works as follow:

1. Fit a CART to the outcome and calculate the (pseudo-) residuals. For a binary outcome this may be the gradient of the Bernoulli Deviance.

2. Fit a new CART to the (pseudo-) residuals using a random split of the data.

3. Shrink the CART via a factor $\lambda$, e.g. 0.001 to slow down the learning 4. Update the ensemble and calculate new (pseudo-) residuals.

5. Iterate steps $2-4$ many times.

The depth of each CART and the number of iterations to use in the expansion was determined by 10 repeats of 10 -fold cross-validation. Cross validation randomly splits the data into $k$ subsets of roughly equal size. The ensemble is fitted to $k-1$ parts and the performance is evaluated on the withheld part. This is repeated for each subset and the $k$ performance estimates are averaged to give a performance estimate for a particular configuration of the CARTs in the ensemble. Cross-validation is a random process in which results may vary depending on how the data are split. To mitigate this effect, the entire process was repeated 10 times and the performance estimates were averaged. The optimal number of CARTs ( 1 through 15,000) and their depths (1-4) were chosen as the combination that minimized the cross-validated Bernoulli deviance.

The interpretability of single CARTs is lost in an ensemble, but variable importance measures and partial dependency plots are two tools to interpret the ensemble. The variable importance was determined by randomly permuting each variable at a time and measures the associated drop in predictive performance. An important variable, which participates in many splits throughout the ensemble, would have a large effect on the predictive power while an unimportant variable would only have a minor effect on the predictive power. As the variable 
importance measure has no absolute relevance, it can be used to determine amongst a group of variables, which are likely to be most predictive, and is scaled so that the variable importance estimates for all variables sum to 100 .

Since an ensemble of CARTs does not provide regression coefficients, partial dependence plot is a graphical way of understanding the nature of the dependencies. The ensemble is a complex function depending on all variables in the data. The partial dependence on a subset of variables in then defined as the predictions for values of the subset averaged over all other data.

Partial dependencies are also used in the evaluation of interaction effects from an ensemble of CARTs. A function $F\left(x_{i}, x_{j}\right)$ is said to be additive if we can write $F\left(x_{i}, x_{j}\right)=F_{i}\left(x_{i}\right)+F_{j}\left(x_{j}\right)$. Friedman and Popescu defined the $H$-statistic as a measure of the additivity of a complex function from an ensemble of depth 2 or deeper CARTs (Friedman and Popescu, 2008). $H$ ranges from 0 to 1 with 0 means no interactions. A sample based estimate of $H$ is never exactly zero and a resampling based method can be used to obtain a null distribution for $H$. An observed value of $H$ far from the null distribution could indicate substantial interactions. CARTs, gradient boosting and the application to the study of mixture effects are described in detail elsewhere (Friedman, 2001; Hastie et al., 2009; Lampa et al., 2014).

Thirty environmental contaminants, sex (age same in all subjects), baseline values of the variables that constitutes the metabolic syndrome, LDL-cholesterol, smoking, education, exercise habits, alcohol, energy and dietary fat intake were evaluated in the model.

The Odds Ratios given in the results were estimated by predicting the response on the logit scale at the 10th and 90th percentiles of the variables and anti-logging the difference. 95\% bias-corrected and accelerated (BCa) confidence intervals were obtained using the bootstrap (Efron and Tibshirani, 1994; Davison and Hinkley, 1997) with 10,000 replicates. Confidence intervals from boosted tree ensembles have to our knowledge not been well studied. Goeman points out that standard errors resulting from penalized estimation are not very meaningful as penalized estimation introduces bias in the estimates to lower their variance (Goeman et al., 2016). The bootstrap quantifies the variance part of the mean-squared error, but an estimate of the bias is impossible to obtain in many situations when penalized estimation is used. The presented intervals are thus confidence intervals for the predicted Odds Ratios in these data rather than confidence intervals for the population Odds Ratios. All analyses were performed using R (R Core Team, 2016) version 3.2.4 using the gbm and boot packages (Hickey et al., 2016; Canty and Ripley, 2017).

\section{Results}

Basic characteristics regarding cardiovascular risk factors are presented in Table 1.

A more detailed description of the measurement of the life-style factors are given in the methods Section 2.4.

Results of the measurements of the 30 environmental contaminants at age 70 are shown in Table 2.

During the 10-year follow-up period, 92 incident cases of the MetS occurred.

Based on the cross-validation (see below), the Bernoulli Deviance was minimized by using 11,502 depth 1 CARTs, i.e. no interactions were modeled. The cross-validated C-index was 0.74 .

Table 3 below shows the relative importance of the investigated variables regarding the association vs. incident MetS. The top variable was HDL-cholesterol, followed by two other variables included in the MetS definition, serum triglycerides and waist circumference. The most important environmental contaminants were PCB126, followed by PCB170, HCB and PCB118.

Of the environmental contaminants studied, PCB126, PCB170, HCB and PCB118 were all associated with incident MetS (OR 1.57 for a change from 10th to 90th percentile (95\%CI 1.07-2.28) for PCB126, OR
Table 3

Relative importance of environmental contaminants, metabolic syndrome variables and some lifestyle factors.

\begin{tabular}{|c|c|}
\hline Variable & Relative importance \\
\hline HDL-cholesterol & 22.8 \\
\hline Serum triglycerides & 20.1 \\
\hline Waist circumference & 10.8 \\
\hline PCB $126^{\mathrm{a}}$ & 7.77 \\
\hline Energy intake & 5.6 \\
\hline РСB 170 & 4.1 \\
\hline HCB & 4.0 \\
\hline Systolic blood pressure & 3.1 \\
\hline PCB 118 & 2.1 \\
\hline $\mathrm{Cd}$ & 1.6 \\
\hline Co & 1.5 \\
\hline Dietary fat intake & 1.5 \\
\hline $\mathrm{PFH} \times \mathrm{S}$ & 1.1 \\
\hline Alcohol intake & 1.1 \\
\hline PCB 209 & 1.0 \\
\hline PCB 169 & 0.9 \\
\hline $\mathrm{Cu}$ & 0.8 \\
\hline OCDD & 0.8 \\
\hline $\mathrm{Pb}$ & 0.8 \\
\hline $\mathrm{Al}$ & 0.8 \\
\hline $\mathrm{Hg}$ & 0.7 \\
\hline Fasting glucose & 0.6 \\
\hline TNC & 0.6 \\
\hline $\mathrm{Ni}$ & 0.6 \\
\hline Sex & 0.5 \\
\hline LDL-cholesterol & 0.5 \\
\hline $\mathrm{p}, \mathrm{p}^{\prime}-\mathrm{DDE}$ & 0.5 \\
\hline LPFOS & 0.4 \\
\hline PFNA & 0.3 \\
\hline PFOSA & 0.3 \\
\hline РCB 153 & 0.3 \\
\hline BDE-47 & 0.3 \\
\hline Mo & 0.3 \\
\hline $\mathrm{Zn}$ & 0.3 \\
\hline PFOA & 0.3 \\
\hline PFDA & 0.2 \\
\hline PFHpA & 0.2 \\
\hline Education & 0.1 \\
\hline $\mathrm{Cr}$ & 0.1 \\
\hline Exercise habits & 0.1 \\
\hline $\mathrm{Mn}$ & 0.1 \\
\hline PFUnDA & 0.1 \\
\hline Smoking & $<0.1$ \\
\hline
\end{tabular}

${ }^{a}$ For abbreviations of chemicals listed in this table please see Table 2 above.

$0.68(0.53-0.87)$ for PCB170, OR 1.38 (1.06-1.91) for HCB and OR 1.36 (1.05-1.94) for PCB118, respectively, in an additive fashion. This model also included HDL- and LDL-cholesterol and serum triglycerides in order to adjust for lipid levels. Please see Fig. 1 below for details.

Fig. 2 below shows the marginal probabilities (partial dependencies) for the ten most important variables for predicting incident MetS.

\section{Discussion}

The present study, using a statistical method previously deemed to be suitable for the study of mixture effects of environmental contaminants (Lampa et al., 2014), showed that a mixture of contaminants, with PCB126, PCB170, HCB and PCB118 being the most important, were related to the development of the MetS in the prospective setting. This is the first large prospective study to investigate the role of a mixture of environmental contaminants on incident MetS.

PCBs have previously been linked to the MetS and its components in cross-sectional studies (Lee et al., 2007; Lind et al., 2013). In the present prospective setting it was mainly the two PCB congeners 126 and 118 that were related to incident MetS independently of each other and in a positive additive fashion. We have previously shown that certain 


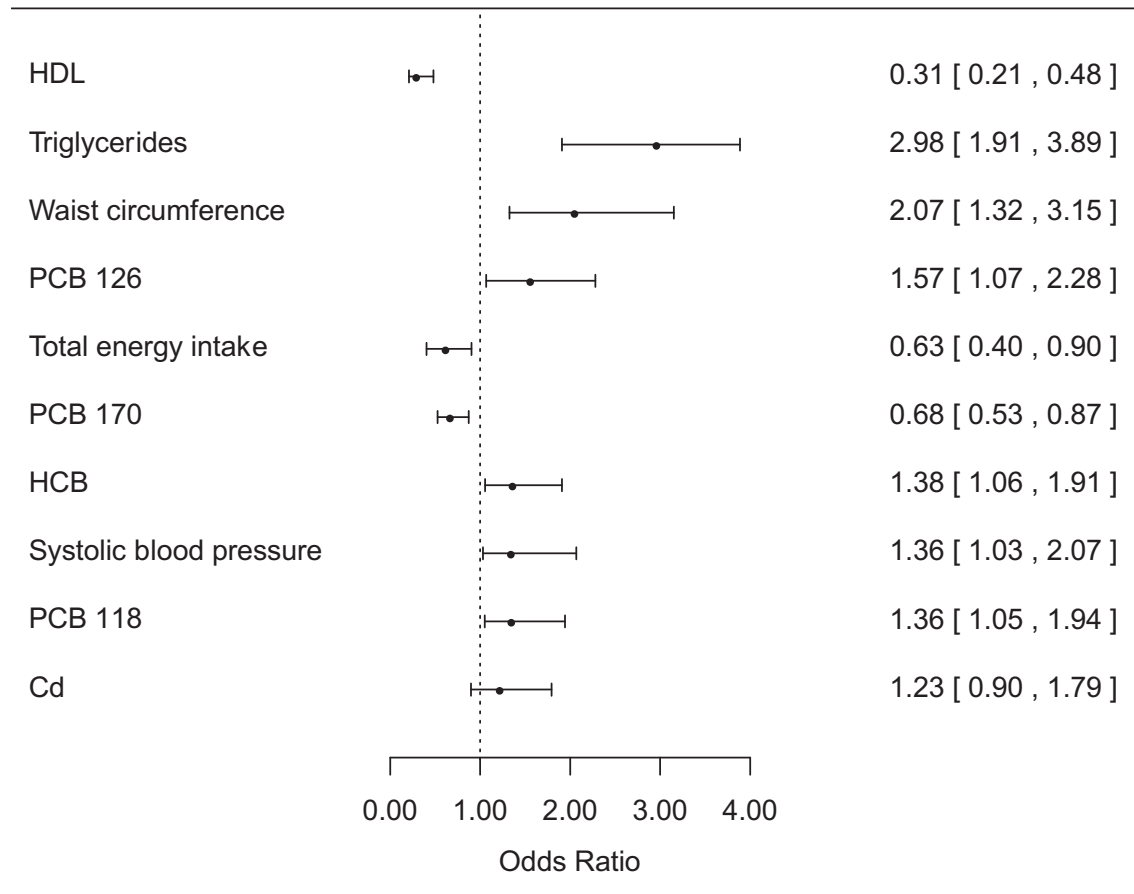

Fig. 1. Predicted effects on incident MetS for the top ten ranked variables. Odds ratios (OR) for a change from 10th to 90th percentile and bootstrapped confidence intervals are shown.
PCBs cluster together, both in the PIVUS study and in the NHANES study (Lampa et al., 2012). Since the $\mathrm{R}^{2}$-value for the relationship between PCB118 and PCB126 only was 0.05, no close relationship between those two PCB congeners was found. It should be noted however that PCB126 is a coplanar non-ortho substituted PCB with a high affinity to the Ah-receptor and that PCB118 is a mono-ortho substituted PCB having a substantially lower affinity to the same receptor. Thus, it might be that the additive effect found for these two PCB congeners is mediated by activation of the Ah-receptor, a main binding site for dioxins. In the cross-sectional setting it has been shown that the TEQ (toxic equivalent), a summary measure of the toxic equivalence factors (TEF) of dioxins, dioxin-like PCBs and furans, was linked to prevalent MetS (Uemura et al., 2009), supporting a role for Ah-receptor binding contaminants, like PCB118 and PCB126 also in the present prospective study. That assumption is supported by a recent study showing that directly measured Ah-receptor binding of sera was higher in subjects with the MetS than in controls (Park et al., 2010).

Amongst the dioxins only OCDD showed measurable levels in the majority of the population, and was therefore the only dioxin included in the present analyses. It should be remembered however that OCDD is a very weak Ah-receptor ligand, a fact that could explain the absence of effect of OCDD in the present study.

In contrast to the positive relationships found between PCB126 and PCB118 and incident MetS, an inverse relationship was seen for PCB170. We have previously shown that PCB170 and other highly chlorinated PCBs (substituted with 6-10 chlorine atoms) are inversely related to obesity in the PIVUS study, either as evaluated by body fat at Dual-energy X-ray absorptiometry (Ronn et al., 2011), or as waist circumference (Lee et al., 2012). Since abdominal obesity is thought to be a key driver of the MetS (Alberti et al., 2006), it was not surprising that PCB170 levels were inversely related also to incident MetS. Although it cannot be excluded that this PCB might have actions that are opposite of the less chlorinated PCBs (substituted with 4-5 chlorine atoms), it seems more likely that this inverse relationship is due to the high lipophilicity of this highly chlorinated compound. As shown by Wolff et al. (2007), circulating levels of POPs will show an inverse relationship with obesity for a substantial number of years following the time of main exposure due to the accumulation in fat tissue. This period will be longer for the highly chlorinated PCBs due to their longer half-life. Any recurrent exposure will also prolong the period during which the relationship between the specific POP and obesity will be negative. Despite the fact that all PCBs were subjected to the same ban back in the 1970s in Sweden, the exposure to the highly chlorinated PCBs with long half-lives is more likely to be prolonged when compared to less chlorinated PCBs with shorter half-lives. Thus, it is likely that the observed negative relationship between PCB170 is due to the kinetic properties of this contaminant. An inverse relationship between highly chlorinated PCBs and obesity has also been documented by other investigators (Dirinck et al., 2011).

HCB had widely been used as a fungicide before it was banned. HCB has been shown to be a weak agonist of the Ah-receptor (Hahn et al., 1989), but also to have effects on sex hormones by increasing estradiol and prolactin and reducing progesterone levels in rats (Pena et al., 2012). It is not known whether it is the Ah-receptor binding of HCB, or other mechanisms of action of HCB, that links this pesticide to incident MetS in the present study. It should be noted that a previous casecontrol study did not find any association between HCB levels and prevalent MetS (Park et al., 2010).

In the present study, we used a previously validated statistical method to investigate the role of mixtures of environmental contaminants, gradient-boosted CARTs (Lampa et al., 2014). Unlike the classical statistical methods that could be used in the present setting, like logistic regression and Cox proportional hazard analysis, gradientboosted CARTs could evaluate both additive and synergistic effects for a large number of exposures in samples sizes as in the present study. Several other methods, like weighted quantile sum (WQS) regression, elastic net, and least absolute shrinkage and selection operator (LASSO), have been proposed for the study of mixture effects (Braun et al., 2016; Taylor et al., 2016). Every technique has its pro and cons, but we have found gradient-boosted regression trees (CARTs) to be a good method for the study of mixture effects in humans, because of the possibility of incorporating both additive and interaction effects (Lampa et al., 2014).

Traditionally, circulating levels of classical POPs are normalized for lipids due to the fact that these compounds are lipid soluble (Bernert et al., 2007). It is however not appropriate to normalize for lipids when dealing with outcomes like the MetS including lipid components, since POP exposure has been shown to alter lipid levels in both humans and animal studies (Bell et al., 1994; Lind et al., 2004; Goncharov, Haase et al., 2008; Kanagawa et al., 2008; Penell et al., 2014). We therefore 

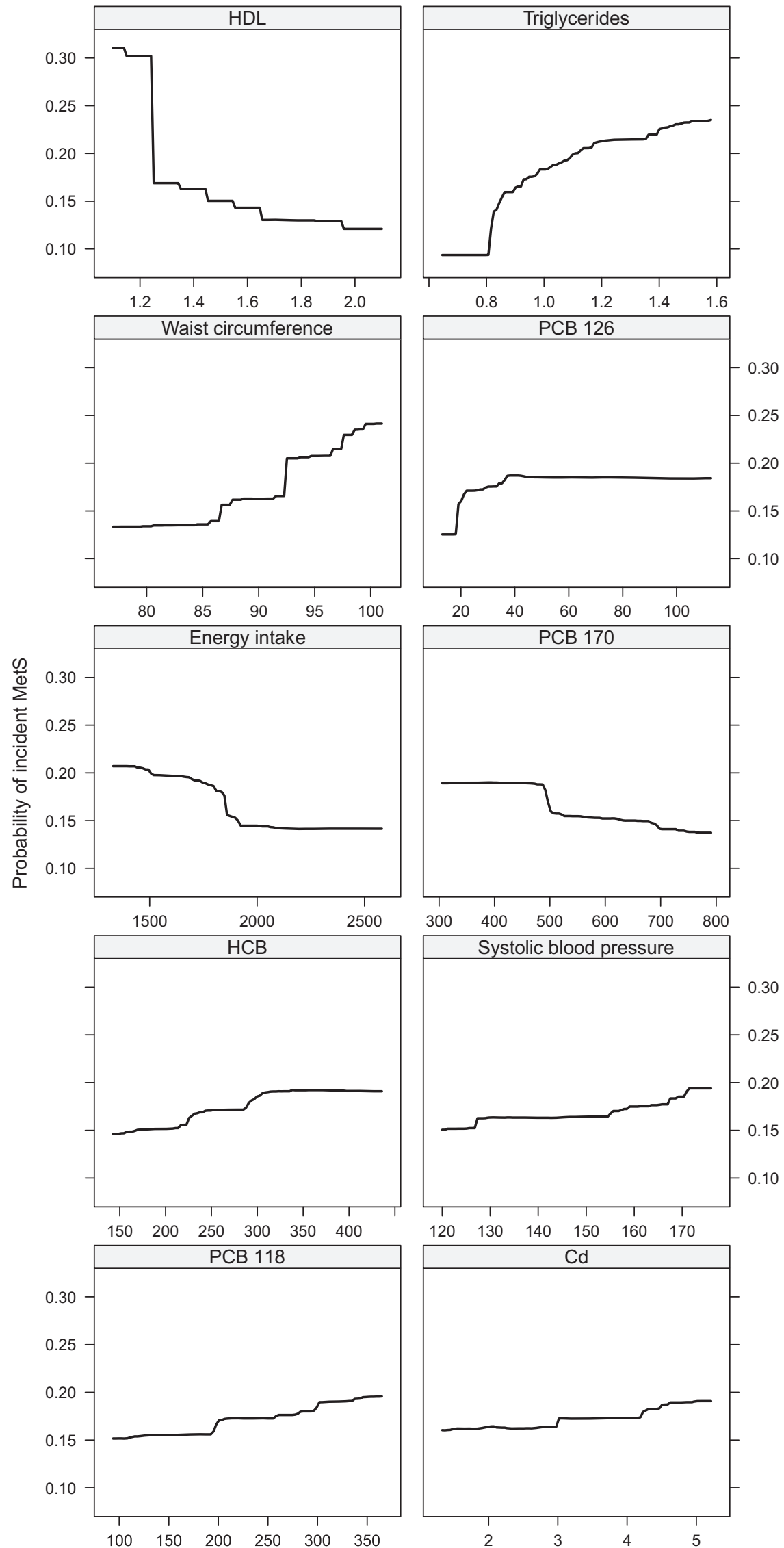
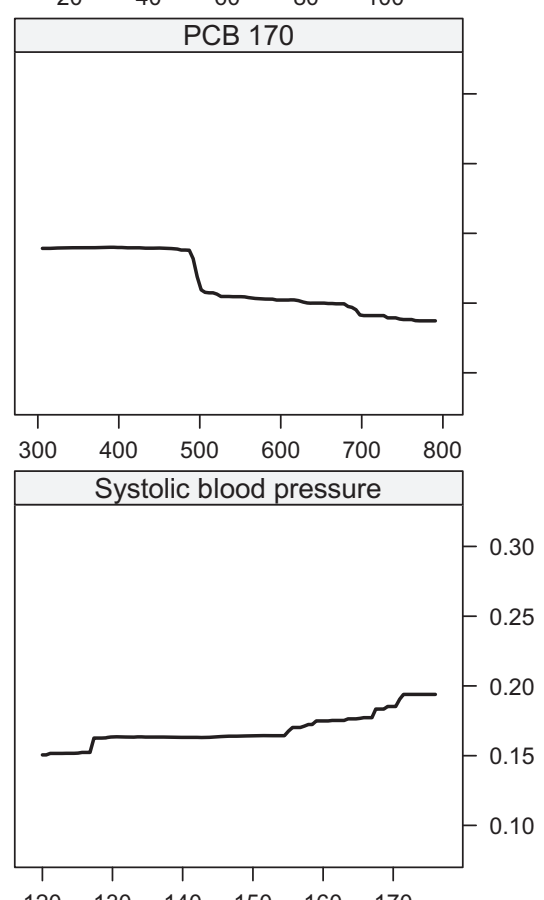

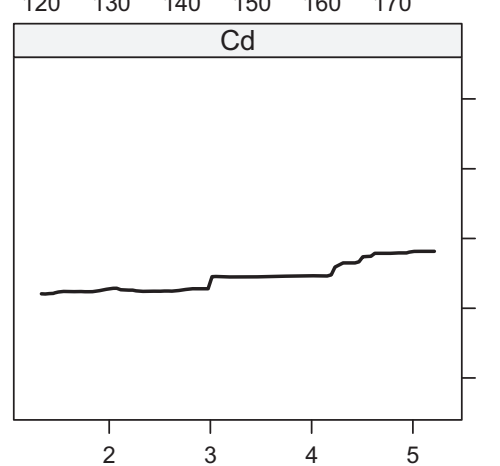

Fig. 2. Marginal probabilities (partial dependencies) for the ten most important variables for predicting incident MetS. The horizontal axes are truncated at the 10th and 90th percentiles of each variable's distribution. The relationships are mainly flat beyond those points. 
used wet-weight levels for the POPs, but adjusted for lipids in the statistical model. This is supported by statistical simulation data showing that adjustment for lipids in the models usually are preferably to lipidnormalization (Gaskins and Schisterman, 2009).

Several different definitions of the MetS have been proposed. We have used the NCEP/ATPIII-criteria, since this definition is by far the most commonly used in research and we have previously validated this definition in terms of mortality (Sundstrom et al., 2006). Furthermore, the few other studies on environmental contaminants and MetS (references 16-19) have used that definition, so we think it is appropriate to use the same definition in order to keep the literature comparable.

In the present study, we found self-reported energy intake to be inversely related to incident MetS. There is a rich literature on underreporting of energy intake in obese subjects, which probably explains the present counter-intuitive finding (Braam et al., 1998).

\subsection{Strength and limitations}

Amongst the strengths of the present study is its prospective design with a reasonably long follow-up period with a fair number of incident cases of the MetS, together with baseline measurements of a large number of environmental contaminants and the application of validated statistical methods.

Amongst the weaknesses is the lack of a full dioxin spectra, which limits our conclusion regarding dioxins and TEQ/Ah-receptor binding as a mechanism behind the associations between some PCBs and incident MetS.

It should also be acknowledged that the present study was conducted in elderly Scandinavians, limiting the generalizability to other age and ethnic groups, and we cannot test the hypothesis regarding fetal exposure, since this cohort was born in the early 1930s when most of the measured chemicals were not used.

Plasma/serum/whole blood is convenient to use in large scale epidemiology. However, for the lipid-soluble POPs fat samples would probable give a better measure of exposure. PFASs are mainly bound to proteins and accumulate in the liver. Some of the metals are accumulating in other tissues than whole blood, for example cadmium accumulation in bone tissue. So for experimental studies or small scale human studies other compartments than blood might give better measures of exposure, but in large scale studies, such as PIVUS, measurements in the circulation, or in urine, is the only realistic way to obtain multiple measurements.

The power to detect any interactions was limited by to the low number of incident cases. CARTs do however emphasize interactions in their fitting and are notoriously poor at fitting additive relationships. Several tree depths were allowed in the parameter tuning process and since depth one CARTs clearly outperformed the rest we believe that any interactions present, despite the algorithm not finding them, are small compared to the additive effects. Larger studies would be needed to confirm these findings.

\section{Conclusions}

In conclusion, of 30 evaluated environmental contaminants, a mixture of contaminants, including PCB126, PCB170, HCB and PCB118, showed associations with future development of the MetS in an additive fashion in this prospective study.

\section{Conflict of interest}

The authors declare no competing financial interests.

\section{Acknowledgments}

We acknowledge all the individuals who participated in this study. This study was supported by the Swedish Research Council for
Environment, Agricultural Sciences and Spatial Planning (FORMAS, grant number 2007-2047).

\section{References}

Administration, S.N.F., 1994. Befolkningens kostvanor och näringsintag i Sverige 1989: metod- och resultatanalys. Swedish National Food Administration (NFA).

Alberti, K.G., Zimmet, P., Shaw, J., 2006. Metabolic syndrome-a new world-wide definition. A Consensus Statement from the International Diabetes Federation. Diabet. Med. 23 (5), 469-480.

Becker, W., 2001. Comparability of household and individual food consumption data-evidence from Sweden. Public Health Nutr. 4 (5b), 1177-1182.

Bell, F.P., Iverson, F., Arnold, D., Vidmar, T.J., 1994. Long-term effects of Aroclor 1254 (PCBs) on plasma lipid and carnitine concentrations in rhesus monkey. Toxicology 89 (2), 139-153.

Bernert, J.T., Turner, W.E., Patterson Jr., D.G., Needham, L.L., 2007. Calculation of serum "total lipid" concentrations for the adjustment of persistent organohalogen toxicant measurements in human samples. Chemosphere 68 (5), 824-831.

Braam, L.A., Ocke, M.C., Bueno-de-Mesquita, H.B., Seidell, J.C., 1998. Determinants of obesity-related underreporting of energy intake. Am. J. Epidemiol. 147 (11), 1081-1086.

Braun, J.M., Gennings, C., Hauser, R., Webster, T.F., 2016. What can epidemiological studies tell us about the impact of chemical mixtures on human health? Environ. Health Perspect. 124 (1), A6-A9.

Canty, A., Ripley, B., 2017. boot: Bootstrap R (S-Plus) Functions.

Carlsson, L., Lind, L., Larsson, A., 2010. Reference values for 27 clinical chemistry tests in 70-year-old males and females. Gerontology 56 (3), 259-265.

Davison, A.C., Hinkley, D.V., 1997. Bootstrap Methods and their Application. Press, Cambridge University.

Dirinck, E., Jorens, P.G., Covaci, A., Geens, T., Roosens, L., Neels, H., Mertens, I., Van Gaal, L., 2011. Obesity and persistent organic pollutants: possible obesogenic effect of organochlorine pesticides and polychlorinated biphenyls. Obesity (Silver Spring) 19 (4), 709-714.

Efron, B., Tibshirani, R.J., 1994. An Introduction to the Bootstrap. Chapman \& Hall/CRC.

Fisher, M., Arbuckle, T.E., Wade, M., Haines, D.A., 2013. Do perfluoroalkyl substances affect metabolic function and plasma lipids?-Analysis of the 2007-2009, Canadian Health Measures Survey (CHMS) Cycle 1. Environ. Res. 121, 95-103.

Friedman, J.H., 2001. Greedy function approximation: a gradient boosting machine. Ann. Stat. 29 (5) (1189-1112-1132).

Friedman, J.H., Popescu, B.E., 2008. Predictive Learning Via Rule Ensembles. pp. 916-954.

Gaskins, A.J., Schisterman, E.F., 2009. The effect of lipid adjustment on the analysis of environmental contaminants and the outcome of human health risks. Methods Mol. Biol. 580, 371-381.

Goeman, J., Meijer, R., Chaturvedi, N., 2016. L1 and L2 Penalized Regression Models, R.

Goncharov, A., Haase, R.F., Santiago-Rivera, A., Morse, G., E. Akwesasne Task Force on the, McCaffrey, R.J., Rej, R., Carpenter, D.O., 2008. High serum PCBs are associated with elevation of serum lipids and cardiovascular disease in a Native American population. Environ. Res. 106 (2), 226-239.

Hahn, M.E., Goldstein, J.A., Linko, P., Gasiewicz, T.A., 1989. Interaction of hexachlorobenzene with the receptor for 2,3,7,8-tetrachlorodibenzo-p-dioxin in vitro and in vivo. Evidence that hexachlorobenzene is a weak Ah receptor agonist. Arch. Biochem. Biophys. 270 (1), 344-355.

Hastie, T., Tibshirani, R., Friedman, J.H., 2009. The Elements of Statistical Learning. Springer-Verlag, New York.

Hickey, J., Metcalfe, P., Ridgeway, G., Schroedl, S., Southworth, H., Therneau, T., 2016 Generalized Boosted Regression Models. R Package Version 2.2, R.

Kanagawa, Y., Matsumoto, S., Koike, S., Tajima, B., Fukiwake, N., Shibata, S., Uchi, H., Furue, M., Imamura, T., 2008. Association of clinical findings in Yusho patients with serum concentrations of polychlorinated biphenyls, polychlorinated quarterphenyls and 2,3,4,7,8-pentachlorodibenzofuran more than 30 years after the poisoning event. Environ. Health 7, 47.

Lampa, E., Lind, L., Hermansson, A.B., Salihovic, S., van Bavel, B., Lind, P.M., 2012. An investigation of the co-variation in circulating levels of a large number of environmental contaminants. J. Expo. Sci. Environ. Epidemiol. 22 (5), 476-482.

Lampa, E., Lind, L., Lind, P.M., Bornefalk-Hermansson, A., 2014. The identification of complex interactions in epidemiology and toxicology: a simulation study of boosted regression trees. Environ. Health 13, 57.

Lee, D.H., Lee, I.K., Porta, M., Steffes, M., Jacobs Jr., D.R., 2007. Relationship between serum concentrations of persistent organic pollutants and the prevalence of metabolic syndrome among non-diabetic adults: results from the National Health and Nutrition Examination Survey 1999-2002. Diabetologia 50 (9), 1841-1851.

Lee, D.H., Lind, P.M., Jacobs Jr., D.R., Salihovic, S., van Bavel, B., Lind, L., 2011. Polychlorinated biphenyls and organochlorine pesticides in plasma predict development of type 2 diabetes in the elderly: the prospective investigation of the vasculature in Uppsala Seniors (PIVUS) study. Diabetes Care 34 (8), 1778-1784.

Lee, D.H., Lind, L., Jacobs Jr., D.R., Salihovic, S., van Bavel, B., Lind, P.M., 2012. Associations of persistent organic pollutants with abdominal obesity in the elderly: the Prospective Investigation of the Vasculature in Uppsala Seniors (PIVUS) study. Environ. Int. 40, 170-178.

Lind, L., Lind, P.M., 2012. Can persistent organic pollutants and plastic-associated chemicals cause cardiovascular disease? J. Intern. Med. 271 (6), 537-553.

Lind, L., Jakobsson, S., Lithell, H., Wengle, B., Ljunghall, S., 1988. Relation of serum calcium concentration to metabolic risk factors for cardiovascular disease. BMJ 297 
(6654), 960-963.

Lind, P.M., Orberg, J., Edlund, U.B., Sjoblom, L., Lind, L., 2004. The dioxin-like pollutant PCB $126\left(3,3^{\prime}, 4,4^{\prime}, 5\right.$-pentachlorobiphenyl) affects risk factors for cardiovascular disease in female rats. Toxicol. Lett. 150 (3), 293-299.

Lind, L., Fors, N., Hall, J., Marttala, K., Stenborg, A., 2005. A comparison of three different methods to evaluate endothelium-dependent vasodilation in the elderly: the Prospective Investigation of the Vasculature in Uppsala Seniors (PIVUS) study. Arterioscler. Thromb. Vasc. Biol. 25 (11), 2368-2375.

Lind, P.M., Zethelius, B., Lind, L., 2012. Circulating levels of phthalate metabolites are associated with prevalent diabetes in the elderly. Diabetes Care 35 (7), 1519-1524.

Lind, P.M., Riserus, U., Salihovic, S., Bavel, B., Lind, L., 2013. An environmental wide association study (EWAS) approach to the metabolic syndrome. Environ. Int. 55, 1-8.

Lind, L., Zethelius, B., Salihovic, S., van Bavel, B., Lind, P.M., 2014a. Circulating levels of perfluoroalkyl substances and prevalent diabetes in the elderly. Diabetologia 57 (3), 473-479.

Lind, P.M., Penell, J., Salihovic, S., van Bavel, B., Lind, L., 2014b. Circulating levels of p, $\mathrm{p}^{\prime}$-DDE are related to prevalent hypertension in the elderly. Environ. Res. 129, $27-31$.

Lind, L., Lind, P.M., Lejonklou, M.H., Dunder, L., Bergman, A., Guerrero-Bosagna, C., Lampa, E., Lee, H.K., Legler, J., Nadal, A., Pak, Y.K., Phipps, R.P., Vandenberg, L.N., Zalko, D., Agerstrand, M., Oberg, M., Blumberg, B., Heindel, J.J., Birnbaum, L.S., 2016. Uppsala consensus statement on environmental contaminants and the global obesity epidemic. Environ. Health Perspect. 124 (5), A81-A83.

Park, S.K., Son, H.K., Lee, S.K., Kang, J.H., Chang, Y.S., Jacobs, D.R., Lee, D.H., 2010. Relationship between serum concentrations of organochlorine pesticides and metabolic syndrome among non-diabetic adults. J. Prev. Med. Public Health 43 (1), 1-8.

Patel, C.J., Cullen, M.R., Ioannidis, J.P., Butte, A.J., 2012. Systematic evaluation of environmental factors: persistent pollutants and nutrients correlated with serum lipid levels. Int. J. Epidemiol. 41 (3), 828-843.

Pena, D., Pontillo, C., Garcia, M.A., Cocca, C., Alvarez, L., Chiappini, F., Bourguignon, N., Frahm, I., Bergoc, R., Kleiman de Pisarev, D., Randi, A., 2012. Alterations in c-Src/ HER1 and estrogen receptor alpha signaling pathways in mammary gland and tumors of hexachlorobenzene-treated rats. Toxicology 293 (1-3), 68-77.

Penell, J., Lind, L., Salihovic, S., van Bavel, B., Lind, P.M., 2014. Persistent organic pollutants are related to the change in circulating lipid levels during a 5 year follow-up. Environ. Res. 134, 190-197.

\section{R Core Team, 2016. R: A language and environment for statistical computing. R}

Foundation for Statistical Computing, Vienna, Austria. https://www.R-project.org/.

Reaven, G.M., 1988. Banting lecture 1988. Role of insulin resistance in human disease Diabetes 37 (12), 1595-1607.

Rodushkin, I., Olofsson, R., Axelsson, M.D., 2000. Determination of 60 elements in whole blood by sector field inductively coupled plasma mass spectrometry. J. Anal. At. Spectrom. 15 (8), 937-944.

Ronn, M., Lind, L., van Bavel, B., Salihovic, S., Michaelsson, K., Lind, P.M., 2011. Circulating levels of persistent organic pollutants associate in divergent ways to fat mass measured by DXA in humans. Chemosphere 85 (3), 335-343.

Salihovic, S., Mattioli, L., Lindstrom, G., Lind, L., Lind, P.M., van Bavel, B., 2012. A rapid method for screening of the Stockholm Convention POPs in small amounts of human plasma using SPE and HRGC/HRMS. Chemosphere 86 (7), 747-753.

Salihovic, S., Karrman, A., Lindstrom, G., Lind, P.M., Lind, L., van Bavel, B., 2013. A rapid method for the determination of perfluoroalkyl substances including structural isomers of perfluorooctane sulfonic acid in human serum using 96-well plates and column-switching ultra-high performance liquid chromatography tandem mass spectrometry. J. Chromatogr. A 1305, 164-170.

Salihovic, S., Karrman, A., Lind, L., Lind, P.M., Lindstrom, G., van Bavel, B., 2015. Perfluoroalkyl substances (PFAS) including structural PFOS isomers in plasma from elderly men and women from Sweden: results from the Prospective Investigation of the Vasculature in Uppsala Seniors (PIVUS). Environ. Int. 82, 21-27.

Sandau, C.D., Sjodin, A., Davis, M.D., Barr, J.R., Maggio, V.L., Waterman, A.L., Preston, K.E., Preau Jr., J.L., Barr, D.B., Needham, L.L., Patterson Jr., D.G., 2003. Comprehensive solid-phase extraction method for persistent organic pollutants. Validation and application to the analysis of persistent chlorinated pesticides. Anal. Chem. 75 (1), 71-77.

Sundstrom, J., Riserus, U., Byberg, L., Zethelius, B., Lithell, H., Lind, L., 2006. Clinical value of the metabolic syndrome for long term prediction of total and cardiovascular mortality: prospective, population based cohort study. BMJ 332 (7546), 878-882.

Taylor, K.W., Joubert, B.R., Braun, J.M., Dilworth, C., Gennings, C., Hauser, R., Heindel, J.J., Rider, C.V., Webster, T.F., Carlin, D.J., 2016. Statistical approaches for assessing health effects of environmental chemical mixtures in epidemiology: lessons from an innovative workshop. Environ. Health Perspect. 124 (12), A227-A229.

Teppala, S., Madhavan, S., Shankar, A., 2012. Bisphenol A and metabolic syndrome: results from NHANES. Int. J. Endocrinol. 2012, 598180.

Uemura, H., Arisawa, K., Hiyoshi, M., Kitayama, A., Takami, H., Sawachika, F., Dakeshita, S., Nii, K., Satoh, H., Sumiyoshi, Y., Morinaga, K., Kodama, K., Suzuki, T., Nagai, M., Suzuki, T., 2009. Prevalence of metabolic syndrome associated with body burden levels of dioxin and related compounds among Japan's general population. Environ. Health Perspect. 117 (4), 568-573.

Wolff, M.S., Anderson, H.A., Britton, J.A., Rothman, N., 2007. Pharmacokinetic variability and modern epidemiology-the example of dichlorodiphenyltrichloroethane, body mass index, and birth cohort. Cancer Epidemiol. Biomark. Prev. 16 (10), 1925-1930. 